\title{
Verzicht auf gesetzliche Regelung zum assistierten Suizid
}

Bei einem assistierten Suizid, das heißt der „Beihilfe zur Selbsttötung“, nimmt der Sterbewillige selbstständig eine Substanz zur Selbsttötung ein. Diese kann ihm von einem Angehörigen oder nahestehenden Menschen, einem Arzt oder Sterbehelfer zur Verfügung gestellt werden. In Deutschland ist die Beihilfe zur Selbsttötung vom Grundsatz her nicht strafbar. Die ärztliche Musterberufsordnung der Bundesärztekammer verbietet es jedoch Ärzten, einen assistierten Suizid durchzuführen. Allerdings haben nicht alle Bundesländer diese Musterberufsordnung übernommen, sodass es hier Unterschiede gibt.

Im Frühjahr 2015 eröffnete der Bundestag eine neue Debatte zur Frage, ob die organisierte Suizidhilfe verboten werden soll. Aus ihr könnte bis November 2015 ein neues Gesetz entstehen. Dieses würde sowohl die Suizidhilfe durch Vereine als auch die Suizidhilfe durch kommerzielle Anbieter unter Strafe stellen. Die Meinungen zu dieser zentralen ethischen Frage differieren quer durch die einzelnen Fraktionen. Daher ist bei dieser Debatte der übliche Fraktionszwang aufgehoben; alle Volksvertreter können frei über die bis dahin eingebrachten Vorschläge abstimmen.

1 Die politische Debatte um gesetzliche Regelungen zum assistierten Suizid wurde zu der bedeutendsten der laufenden Legislaturperiode erklärt. Sie fand breite Resonanz in den Medien und der Öffentlichkeit. Kontroversen um ethische Dilemmata erzeugen öffentliche Aufmerksamkeit. Sie dürfen aber nicht mit gesetzgeberischem Handlungsbedarf gleichgesetzt werden.

Wiederholt sind im Deutschen Bundestag Debatten über eine gesetzliche Regelung des assistierten Suizides geführt worden. In ihnen stehen sich sehr unterschiedliche Positionen gegenüber. Die Debatten werden ohne Erwartungen im Hinblick auf Fraktionsdisziplinen geführt. Dies verspricht einen anspruchsvollen Diskurs. Dem Gesetzgeber fällt es allerdings (regelmäßig) schwer, Debatten im Deutschen Bundestag nicht mit dem Ziel zu führen, eine gesetzliche Regelung herbeizuführen. Das scheint auch beim Thema assistierter Suizid der Fall. Es stehen sich unterschiedliche Gesetzesentwürfe gegenüber. Wir meinen: der Verzicht auf eine gesetzliche Regelung wäre weise.

Bei der Debatte um den assistierten Suizid stehen die zentralen Werte unserer modernen Gesellschaft im Vordergrund, die der Selbstbestimmung und Autonomie.

Diese Werte sind unverzichtbar Teil aufgeklärter und moderner Gesellschaften.
Die Vereinseitigung der Wertedebatte zugunsten der Selbstbestimmung passt zu einer individualisierten und auf Leistung hin ausgerichteten Gesellschaft. Insbesondere die mit schweren körperlichen oder psychischen Erkrankungen assoziierten Gefährdungen eines würdevollen, autonomen Lebens stellen sich als Bedrohung für den modernen Menschen dar. Eine gesetzliche Regelung zum assistierten Suizid, die die „Selbstbestimmung“ als Schutz vor würdelosen Lebensbedingungen sichern will, kapituliert vor dem kulturellen und sozialstaatlichen Auftrag, Bedingungen menschenwürdigen und guten Sterbens zu schaffen.

3 Die mit einer Gesellschaft des langen Lebens verbundenen Herausforderungen verlangen nach einer Auseinandersetzung mit Fragen des Menschseins, mit dem Verständnis von Würde und mit den Vorstellungen eines guten und sinnerfüllten Lebens unter Bedingungen der Vulnerabilität.

Vorstellungen von Leben und Autonomie, die den Beziehungscharakter menschlichen Lebens und dessen Verwiesenheit auf andere nicht einbezieht, ist unvollständig. Ein Bild von Würde, das mit persönlicher Leistungsfähigkeit verbunden wird, gefährdet den Respekt vor jenen Menschen, die in erhöhtem Maße vulnerabel sind. Wesentliches Kennzeichen des verfassungsrechtlichen Würdeverständnisses ist, dass Würde nicht verlie- hen, sondern jedem Menschen mitgegeben ist. Eine Debatte über den assistierten Suizid, die von der Sorge um den Würdeverlust von Menschen mit hoher Verletzlichkeit getrieben ist, tangiert das Würdeverständnis (auch unserer Verfassung). Manche Debattenbeiträge sind insofern nicht wirklich würdeverträglich.

4 Es gibt Situationen, in denen menschliches Leid und Schmerzen nicht in der Weise aufgefangen werden können, wie sich die Helfenden und die Patienten es wünschen. Diese Situationen haben viele Menschen vor Augen, wenn sie eine gesetzliche Regelung des assistierten Suizides fordern.

Entsprechende Forderungen an den Gesetzgeber sind ebenso nachvollziehbar wie Forderungen, zur Selbstbestimmung am Lebensende unabhängig von Leidenszuständen auch die Option zu zählen, sich beim Sterben einer Assistenz bedienen zu können. Ganz überwiegend sind Palliativmedizin und Palliative Care in der Lage, Leidenszustände und Symptome so zu lindern, dass der Wunsch, mit Assistenz zu sterben, zurücktritt. Die moderne palliative Medizin ist auch in den Grenzfällen handlungsfähig. Das kann Menschen die Ängste nehmen, die sie zur Option des assistierten Suizides als letzte Rettung greifen lässt. Hochproblematisch erscheint es, wenn eine gesetzliche Regelung so ausgestaltet wird, dass die Entscheidung über den Vollzug des assistierten Suizides zu einem Zeitpunkt getroffen werden muss, der weit vor einem befürchteten Leidenszustand liegt.

Es gehört zu den letzten Gründen für die Notwendigkeit des Rechtes, dass es Handlungen, die verständlich sind, dennoch negierbar macht.

Es gibt keine gute gesetzliche Regelung für den assistierten Suizid. Darin sind sich (fast) alle Experten einig. Eine gesetzliche Regelung würde überdies ein falsches Signal in die Zeit und in die Gesellschaft senden: Es kann und wird 
missverstanden werden, als Aufkündigung der Solidarität mit den Schwachen. Das geltende Recht, das die Beihilfe zum Suizid nicht unter Strafe stellt, wird durch keine der vorgeschlagenen gesetzlichen Regelungen in einer Weise verbessert, die eine gesetzliche Regelung rechtfertigen würde. Zudem ist zu bedenken, dass Gedanken an den Suizid vor allem dann zunehmen, wenn die Angst zunimmt, mehr und mehr aus Beziehungen $\mathrm{zu}$ fallen, keine Ansprache mehr zu finden, eine Belastung für andere Menschen zu sein. Mit diesen Ängsten ist noch einmal in besonderem Maße die Verantwortung sozialer Netzwerke, aber auch unserer Gesellschaft für die Erhaltung von Lebensqualität in den Grenzsituationen menschlichen Lebens angesprochen.

\section{$6 \quad$ Es wäre eine weise Entscheidung des Gesetzgebers, er würde sich}

\section{bewusst dazu durchringen, auf eine gesetzliche Regelung gänzlich zu verzichten.}

Er sollte sie durch eine Entschließung ersetzen, die zentrale kulturelle, fachliche und gesundheitspolitische Herausforderungen betont: die Akzeptanz eines Lebens unter Bedingungen der Vulnerabilität, die Sorgefähigkeit der Gesellschaft und die Integration der Verantwortung für andere in die persönliche Lebensführung als Teil sinnerfüllten Lebens. Auch politisch ist dem Individualismus eine neue Bedeutung des sozialen Miteinanders, der Sorge gegenüberzustellen.

7 Die Liberalität der Gesellschaft, die auch im Zusammenhang mit der Debatte um den assistierten Suizid nicht in Frage gestellt werden darf, muss die Solidarität mit den

\section{Schwachen zum ethischen Prüfstein erklären.}

Eine allein individualethisch geführte Debatte steht in der Gefahr, die Befindlichkeit der Diskurspartner in den Vordergrund zu stellen und sozialethische Dimensionen in gefährlicher Weise zu vernachlässigen. Es waren immer die sozial Schwachen und Vulnerablen, denen notwendige Solidarität versagt wurde und wird: Behandlungsabbrüche, heute bereits eine der häufigsten Todesursachen, treffen in besonderer Weise Menschen mit geringem sozialen Status und geringer sozialen Unterstützung. Die Debatte um den assistierten Suizid ist eine Debatte der Starken. Den Schwachen gehört zuvörderst die politische Aufmerksamkeit.

Prof. Dr. Thomas Klie, Prof. Dr. Andreas Kruse und zahlreiche namhafte Unterstützer. 